\title{
Effect of Misoprostol on Ultrastructural Changes of Renal Tissues in Cisplatin-Treated Adult Rats
}

\section{Ashraf Youssef Nasr*}

Anatomy Department, Faculty of Medicine, Zagazig University, Zagazig, Egypt

\begin{abstract}
Background: Cisplatin (CP) is one of the most effective antineoplastic drugs used for the treatment of various solid tumors. However, the nephrotoxic effect of CP limits its clinical use. The present study aimed to evaluate the possible protective effect of misoprostol (MP) against cisplatin-induced nephrotoxicity.

Material \& Methods: Twenty four male rats were randomly divided into four equal groups (control, cisplatintreated, misoprostol-treated and combined misoprostol and cisplatin-treated). Group II rats were treated with CP single i.p. dose of $7.5 \mathrm{mg} / \mathrm{kg}$ on $5^{\text {th }}$ day. Group III Rats were treated with oral MP at a dose of $200 \mu \mathrm{g} / \mathrm{kg} / \mathrm{day}$ for nine days. Group II rats were treated with CP single i.p. dose of $7.5 \mathrm{mg} / \mathrm{kg}$ day MP for nine consecutive days and injected with CP single i.p. dose $(7.5 \mathrm{mg} / \mathrm{kg})$ on $5^{\text {th }}$ day. Body weight of rats was recorded on $1^{\text {st }}, 3^{\text {rd }}, 5^{\text {th }}, 7^{\text {th }}$ and $9^{\text {th }}$. days. On the $10^{\text {th }}$ day, the rats were anesthetized by ether and scarified. Blood samples were obtained to determine creatinine and BUN levels. Body weight, Kidney weight $\&$ body weight/kidney weight ratio were calculated. Specimens of rats kidneys were prepared for both light and electron microscopic examinations.

Results: single dose of $C P(7.5 \mathrm{mg} / \mathrm{kg}$ i.p) resulted in a significant decrease of body weight $(P .<0.001)$, decrease of kidney weight (P.<0.005), increase of relative kidney weight/body weight ratio $(P .<0.005)$ and increase of creatinine and BUN levels $(P .<0.001)$ compared to control rats. Combination of MP with CP improved most of the previous parameters. In CP-treated rats, marked degenerative and necrotic changes were noticed at the cortico-medullary renal zone. In combined MP and CP-treated rats, mild degenerative changes were seen within the renal tissues.
\end{abstract}

Conclusion: The present study may provide promising results about the protective effect of MP against CPinduced nephrotoxicity that may be of value in tumor therapy programs.

Keywords: Cisplatin; Nephrotoxicity; Misoprostol; Ultrastructure; kidney; Rat

\section{Introduction}

Cisplatin $(\mathrm{CP})$ is a divalent water-soluble inorganic platinumcontaining complex that is widely used in the treatment of various tumors of the different systems of the human body [1]. However, accumulation of CP within the tubular epithelial cells at the coticomedullary zone leads to the development of nephrotoxicity that limits its clinical use [2].

The exact pathophysiological mechanisms implicated in CPinduced nephrotoxicity are still not fully understood [3]. Several such mechanisms have been suggested including lipid peroxidation and oxidative stress with increased production of reactive oxygen species (ROS) [4] and induction of superoxide anion [5].

Several antioxidants have been used to protect against the deleterious effects of $\mathrm{CP}$ on the kidney [4,6,5]. Misoprostol (MP) is a synthetic methylene prostaglandin E1 (PGE1) analogue that is used in the treatment of peptic ulcer [7]. The ROS scavenger effect of MP has gained a considerable interest within the past few years [8]. In addition, MP has antiapoptotic and cytoprotective effects as well $[9,10]$. The effect of MP on CP-induced changes in lipid peroxidation and antioxidant enzymes in rat kidney has been investigated [6]. However, no study was done to clarify the possible protective effect of MP on the CP-induced changes at the ultrastructural level in rat kidney.

The present study was designed to investigate the possible protective effects of MP on $\mathrm{CP}$-induced biochemical and ultrastructural morphological changes in rat kidney.

\section{Materials and Methods}

\section{Animals and drugs}

Twenty-four adult male Wistar albino rats were used in the present study. The rats were obtained from the animal house, Faculty of Medicine, Zagazig University, Zagazig, Egypt. The rats were conditioned for one week at room temperature. The rats were provided with commercial balanced diet, tap water and ad libitum throughout the experimental period. CP (Unistin vial, EIMC, Cairo) and MP (Misotac, SIGMA, Egypt) were used in this study. Each Unistin vial (50 $\mathrm{ml}$ ) contains $50 \mathrm{mg}$ cisplatin ( $1 \mathrm{ml} / 1 \mathrm{mg} \mathrm{CP}$ ). Each tablet of MP contains

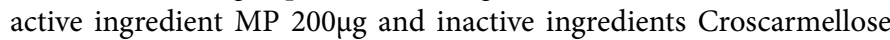
sodium, Aerosil 200, microcrystalline cellulose.

\section{Experimental design}

The rats were randomly divided into four equal groups, six rats each. The rats of each group were housed in a separate plastic cage at a temperature of $25 \pm 2{ }^{\circ} \mathrm{C}$ with a $12 \mathrm{~h}$ light/dark cycles and a relative

*Corresponding author: Ashraf Youssef Nasr, Anatomy Department, Faculty of Medicine, Zagazig University, Zagazig, Egypt, Tel. 002/055/3763530; Fax. 002/055/2307830; E-mail: ashrafnaeem2013@yahoo.com, ashrafnaeem2013@ gmail.com

Received May 25, 2013; Accepted June 11, 2013; Published June 14, 2013

Citation: Nasr AY (2013) Effect of Misoprostol on Ultrastructural Changes of Renal Tissues in Cisplatin-Treated Adult Rats. J Cytol Histol 4: 175. doi:10.4172/21577099.1000175

Copyright: () 2013 Nasr AY. This is an open-access article distributed under the terms of the Creative Commons Attribution License, which permits unrestricted use, distribution, and reproduction in any medium, provided the original author and source are credited. 
humidity of $50-60 \%$. Rats in group I (control) and group II (CP-treated) were given $1 \mathrm{ml}$ of distilled water orally for 9 days and were injected with a single dose of $0.5 \mathrm{ml}$ normal saline intraperitoneal (i.p.) (group I) or a single dose of CP $7.5 \mathrm{mg} / \mathrm{kg}$ body weight i.p. (group II) on the $5^{\text {th }}$ day. Rats in group III (MP-treated) received MP $200 \mu \mathrm{g} / \mathrm{kg}$ body weight orally for 9 consecutive days and were injected with a single dose of $0.5 \mathrm{ml}$ normal saline i.p. on the $5^{\text {th }}$ day. Rats in group IV (MP+CP- treated) were treated with oral dose of MP $200 \mu \mathrm{g} / \mathrm{kg}$ body weight for nine days then were given a single dose of CP $(7.5 \mathrm{mg} / \mathrm{kg}$ BW. i.p. $)$ on the $5^{\text {th }}$ day.

The weight of the rats of all groups were recorded on the $1^{\text {st }}, 3^{\text {rd }}$, $5^{\text {th }}, 7^{\text {th }}$ and $9^{\text {th }}$ days of the study to calculate the initial and final body weights. The rats were anesthetized with ether and sacrificed on the $10^{\text {th }}$ day ( $6^{\text {th. }}$ day of CP injection). Blood samples were collected from the heart by direct intracardiac puncture.

\section{Kidney function assessment}

The blood samples were centrifuged at $3000 \mathrm{rpm}$ for 15 minutes, the serum was separated and used within 48 hours for estimation of the blood urea nitrogen (BUN) and creatinine levels $[11,12]$.

\section{Kidney as ratio of body weight}

The two kidneys of each animal were excised, washed with ice cold normal saline, cleaned from the surrounding fat, dried by filter paper and weighed. The weight of the wet Kidney and its relative weight of wet kidney to the body weight were calculated using the following formula, organ ratio $(\%)=$ organ weight $(\mathrm{gm}) \times 100 /$ body weight $(\mathrm{gm})$.

\section{Light histological examination}

Kidney sections were fixed in $10 \%$ neutral-buffered formalin solution for 48 hours, dehydrated in ascending grades of ethyl alcohol, cleared in xylol and embedded in paraffin wax blocks. 3-5 $\mu \mathrm{m}$ sections were cut using Lieca microtome, washed in water bath and left in the oven for dewaxing. The sections were stained with hematoxylin and eosin (H\&E), Masson's trichrome(MTC), and Periodic acid Schiff's (PAS) [13]. The H\&E stain revealed the general histological architecture of the renal parenchyma. PAS stain was used to determine the concentration of polysaccharides, neutral mucopolysaccharides and glycoproteins within the cellular elements of renal structures, where the kidney sections were incubated with periodic acid for 5 minutes and washed with distilled water. The sections were then incubated with Schiff's reagent for 15 minutes and counter-stained with hematoxylin for 30 seconds. MTC stain was used for demonstration of the collagen connective tissue distribution within the renal structure. The stained tissue slides were mounted with DPX and covered with cover slips to be examined under the light microscope (Nikon E800).

\section{Electron microscopic examination}

From each animal, kidney specimens $\left(1 \mathrm{~mm}^{3}\right)$ were cut and fixed into $3 \%$ glutaraldehyde buffered with $0.1 \mathrm{M}$ phosphate at $4^{\circ} \mathrm{C}$. After rinsing in the buffer, the samples were post-fixed in $1 \%$ osmium tetroxide for $2 \mathrm{hrs}$ at $4^{\circ} \mathrm{C}$. The specimens were washed and dehydrated in ascending grades of ethyl alcohol. Then, the samples were treated with propylene oxide solution and embedded for one hour in a mixture of 1:1 Epon-Araldite. Polymerization was done in the oven at $65^{\circ} \mathrm{C}$ for $48 \mathrm{hrs}$. The kidney sections were cut with a glass knife on LKB-2000S, ultramicrotome, mounted on glass slides and stained with buffered toluidine blue. The appropriate areas were selected with the light microscope. Ultrathin sections $(60-70 \mathrm{~nm})$ were cut with a diamond knife on an LKB ultramicrotome, then mounted on copper grids and double stained with uranyl acetate and lead citrate to be examined under the electron microscope (Joel 100s TEM).

The present study was conducted in accordance with the guidelines for animal research from the National institutes of health and after the approval of the medical ethical committee on animal research at Zagazig University, faculty of medicine, Zagazig, Egypt.

\section{Statistical analysis}

All data were expressed as mean $\pm \mathrm{SD}$. The data were subjected to SPSS software version 16 . The significance of differences between the mean values were calculated using unpaired Student's t-test. $\mathrm{P}$-value $<0.05$ were considered to be statistically significant.

\section{Results}

\section{Effect of CP and / or MP on body weight of rats}

Comparing the initial and final body weights of rats in different groups, a significant weight gain $(\mathrm{P}<0.001)$ was detected in control and MP-treated rats. Conversely, a significant weight loss $(\mathrm{P}<0.001)$ was recorded in the $\mathrm{CP}$-treated rats. Significant difference was observed in the final body weight of the other two groups versus those of the control rats $(\mathrm{P}<0.001)$. Moreover, a significant difference was detected between the final body weight $\mathrm{CP}$-treated and MP-treated rats $(\mathrm{P}<0.001)$. In $\mathrm{MP}+\mathrm{CP}$-treated rats group, a restoration of the body weight loss was observed compared to the cisplatin-treated rats group. Moreover, a significant difference of kidney weight of the CP-treated, MP-treated and MP + CP-treated rats was noticed versus that of the control rats at $\mathrm{P}<0.005)$. The kidney/body weight ratio revealed significant difference $(\mathrm{P}<0.005)$ in both $\mathrm{CP}$-treated and combined MP + CP-treated rats when compared to the control rats (Table 1).

\section{Effect of CP and / or MP on kidney function biomarkers}

Creatinine and BUN levels in the MP-treated rats showed no significant difference when compared to control, whereas an highly significant difference was recorded in creatinine and BUN levels in cisplatin-treated rats $(\mathrm{P}<0.001)$ when compared to control and combined MP + CP-treated rats (Table 2).

\begin{tabular}{|c|c|c|c|c|c|c|}
\hline \multirow{2}{*}{$\begin{array}{c}\text { Animal Group } \\
(n=6)\end{array}$} & \multirow{2}{*}{$\begin{array}{c}\text { Initial Body Weight } \\
(\text { gm) }\end{array}$} & \multirow{2}{*}{$\begin{array}{c}\begin{array}{c}\text { Final Body Weight } \\
\text { (gm) }\end{array} \\
\text { Mean } \pm \text { SD }\end{array}$} & \multicolumn{2}{|c|}{$\begin{array}{l}\text { Change in } \\
\text { Body weight }\end{array}$} & \multirow{2}{*}{$\begin{array}{c}\text { Kidney weight }(\mathrm{gm}) \\
\text { Mean } \pm \text { SD }\end{array}$} & \multirow{2}{*}{$\begin{array}{c}\text { (kidney / Body Weight Ratio) } \\
\text { X } 1000 \\
\text { Mean } \pm \text { SD }\end{array}$} \\
\hline & & & $\operatorname{gm}( \pm)$ & $\%( \pm)$ & & \\
\hline Control & $186.3 \pm 4.6$ & $205.3 \pm 5.8^{a}$ & $(+) 19 \pm 2.8$ & $(+) 10.2 \%$ & $0.8 \pm 0.01$ & $3.9 \pm 0.0003$ \\
\hline Cisplatin & $191.0 \pm 4.7$ & $183.8 \pm 4.3^{\mathrm{ab}}$ & $(-) 7.5 \pm 0.8$ & $(-) 3.9 \%$ & $0.72 \pm 0.014^{d}$ & $4.2 \pm 0.0002^{\mathrm{e}}$ \\
\hline Misoprostol & $184.3 \pm 4.8$ & $193.83 \pm 7.11^{\mathrm{abc}}$ & (+) $9.5 \pm 3.1$ & $(+) 5.2 \%$ & $0.74 \pm 0.014^{d}$ & $3.8 \pm 0.0012$ \\
\hline$M P+C P$ & $185.8 \pm 4.9$ & $186.50 \pm 5.6^{b}$ & (+) $0.8 \pm 1.9$ & $(+) 0.43 \%$ & $0.76 \pm 0.012^{d}$ & $4.0 \pm 0.0002^{\mathrm{e}}$ \\
\hline
\end{tabular}

All data are expressed as Mean $\pm S D, n=6$. a: where $P<0.001$ of initial vs. final $B W$ of same group; $b$ : $P<0.001$ vs. final $B W$ of controls; : $P<0.001$ vs. final $B W$ of cisplatintreated group; d: Significantly different of kidney weight at P.< 0.005$)$ vs. control; e: Significantly different in kidney weight/body weight ratio vs. normal control group at P. $<0.005$. (+) increase. (-): decrease.

Table 1: Effect of cisplatin and / or misoprostol on body weight \& kidney weight/body weight ratio in rats. 
Citation: Nasr AY (2013) Effect of Misoprostol on Ultrastructural Changes of Renal Tissues in Cisplatin-Treated Adult Rats. J Cytol Histol 4: 175. doi:10.4172/2157-7099.1000175

\begin{tabular}{|l|c|c|}
\hline $\begin{array}{l}\text { Animal group } \\
(\mathbf{n}=6)\end{array}$ & Serum Creatinine $(\mathbf{m g} / \mathbf{d l})$ & BUN $(\mathbf{m g} / \mathbf{d l})$ \\
\hline Control & $0.96 \pm 0.08$ & Mean \pm SD \\
\hline Cisplatin & $2.11 \pm 0.11^{\mathrm{a}}$ & $22.3 \pm 3.37$ \\
\hline Misoprostol & $0.95 \pm 0.042^{\mathrm{b}}$ & $58.03 \pm 3.27^{\mathrm{a}}$ \\
\hline Misoprostol + Cisplatin & $1.46 \pm 0.12^{\mathrm{ab}}$ & $24.4 \pm 3.36^{\mathrm{b}}$ \\
\hline
\end{tabular}

All data are expressed as Mean $\pm S D, n=6$. a: Significantly different Vs. contro group at $\mathrm{P}<0.001 . \mathrm{b}$ : $\quad$ Significantly different $V$ s. cisplatin-treated group at $\mathrm{P}$. $<0.001$

Table 2: Effect of cisplatin and/or misoprostol on serum creatinine and BUN (mg/ dl) in Rat.

\section{Light microscopy}

The renal cortex of control rats revealed a normal corpuscular and tubular histological structure (Figure 1A). In CP-treated rats, degenerative changes were markedly apparent within the proximal tubules and to a lesser extent within the distal tubules and renal corpuscles (Figure 1B). The proximal tubules within the deep cortical zone showed degeneration, necrosis and apoptotic nuclear changes of their epithelial cell lining. Luminal dilation with accumulation of homogenous cast and loss of apical microvilli (brush border) was also observed. The distal convoluted tubules revealed luminal dilatation with excessive cast accumulation. The renal corpuscles showed dilated capsular space with condensed glomerular capillary tuft. Peritubular mononuclear inflammatory cell infiltration, vascular congestion and focal hemorrhagic areas were noticed within the renal cortex as well (Figure1B). The renal cortex of the MP-treated rats revealed inflammatory cellular infiltration and vascular congestion (Figure1C). In the MP and CP-treated rats, mild tubular degeneration with luminal dilatation and mononuclear cell infiltration were seen within the renal cortex (Figure 1D).

The renal medulla of control rats showed normal tubular structure
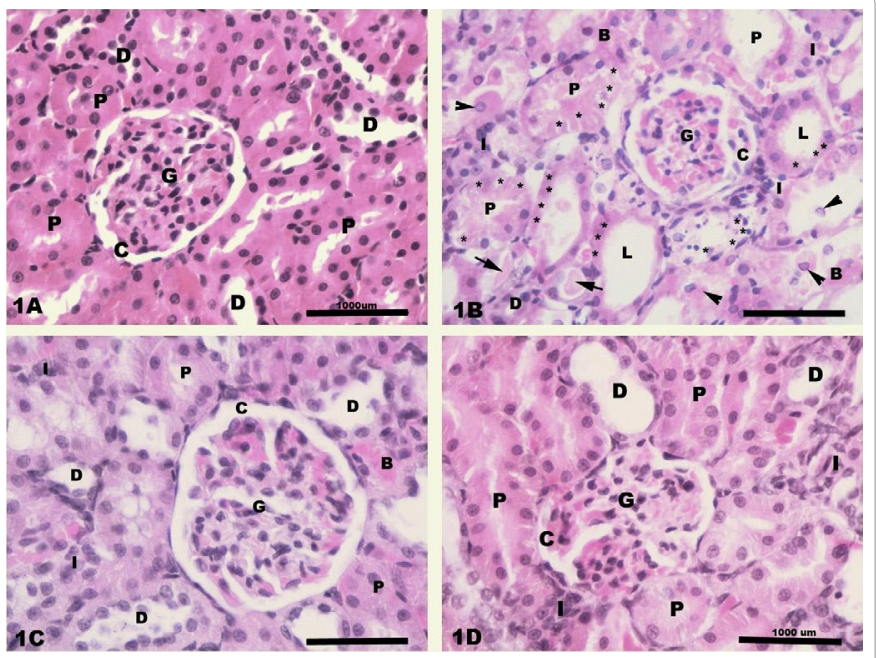

Figure 1: Light photomicrographs of rats renal cortex showing $1 \mathrm{~A})$ normal structure in control rats; $1 \mathrm{~B}$ ) degeneration and necrosis $\left(^{*}\right)$ of the proximal tubular cells $(P)$ in cisplatin-treated rats. Both proximal and distal $(D)$ tubules contain detached epithelial cells (arrow head) and excessive amount of cast (arrow) within their wide lumina (L). The renal corpuscle shows condensation of the glomerular capillary tuft $(\mathrm{G})$ and dilation of the capsular space $(\mathrm{C})$. Peritubular cellular infiltration (I) and congestion (B) are seen as well; $1 \mathrm{C}$ ) The renal cortex shows an interstitial cellular infiltration (I) and congestion (B) in the misoprostoltreated rats; 1D) A few degenerated epithelial cells $\left(^{*}\right)$ are seen in few proximal tubules $(P)$ with peritubular cellular infiltration $(I)$ and congestion $(B)$ in combined misoprostol and cisplatin- treated rats.
(Figure 2A). In CP-treated rats, mild tubular degeneration, peritubular cellular infiltration and congestion, dilated loops of Henle with accumulation of cast were observed within the renal medulla (Figure 2B). In MP-treated rats, peritubular congestion was seen in the renal medulla (Figure 2C). In MP and CP-treated rats, mild dilatation of Henle' loops with cast accumulation and peritubular cellular infiltration were noted in the renal medulla (Figure 2D).
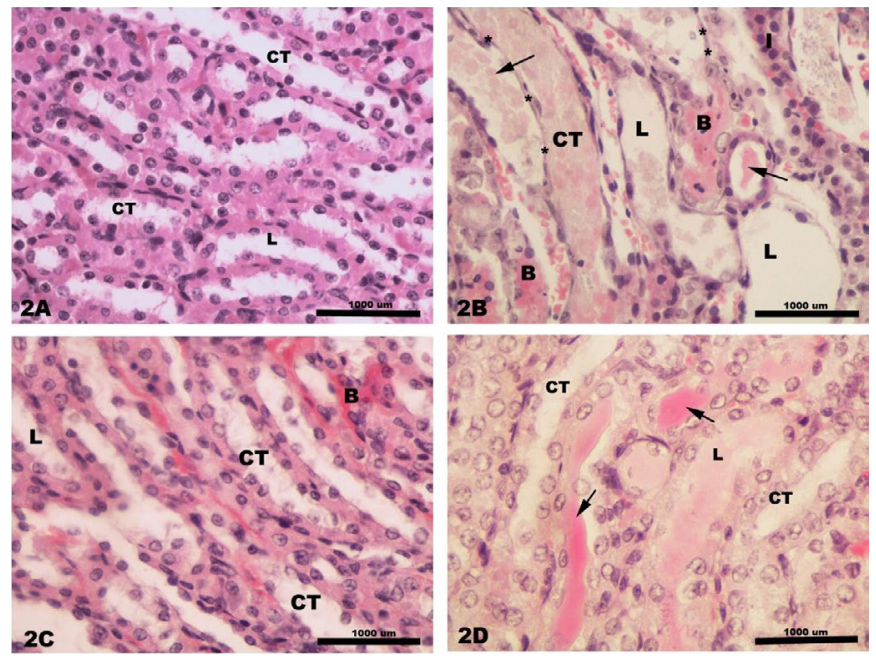

Figure 2: Light photomicrographs of rats renal medulla showing 2 A) the collecting tubules (CT) having normal structure and empty lumen (L) in control rats; $2 \mathrm{~B}$ ) the collecting tubules $(\mathrm{CT})$ have thin necrotic wall $\left(^{*}\right)$ and dilated lumen (L) containing an excessive amount of cast deposition (arrow) in cisplatin-treated rats. Also, peritubular cellular infiltration (I) and congestion (B) are seen within the renal medulla in cisplatin-treated rats as well; $2 \mathrm{C}$ ) the collecting tubules (CT) show normal structure with peritubular congestion $(B)$ in misoprostol-treated rats; 2D) homogenous cast (arrow) is seen within the slightly dilated lumen of the collecting tubules (CT) in combined misoprostol+ cisplatin-treated rats.
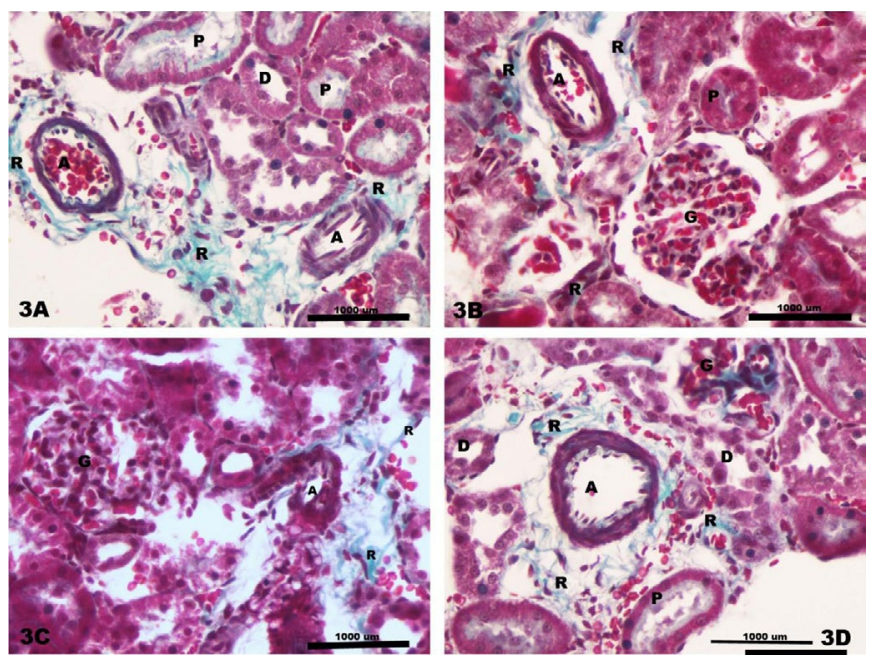

Figure 3: Light photomicrographs of masson trichrome stained sections of rat renal cortex showing little amount of the collagen fibers $(R)$ distributed mainly around the renal vascular structures $(A)$ in control rats $(3 A)$, cisplatin-treated (3B), misoprostol-treated (3C) and combined misoprostol and cisplatin-treated (3D) rats with no noticeable difference.

The control rats showed normal distribution of collagen fibers in the capsular wall, peritubular and around the blood vessels within the renal cortex (Figure 3A). The amount and distribution of the collagen 
Citation: Nasr AY (2013) Effect of Misoprostol on Ultrastructural Changes of Renal Tissues in Cisplatin-Treated Adult Rats. J Cytol Histol 4: 175. doi:10.4172/2157-7099.1000175
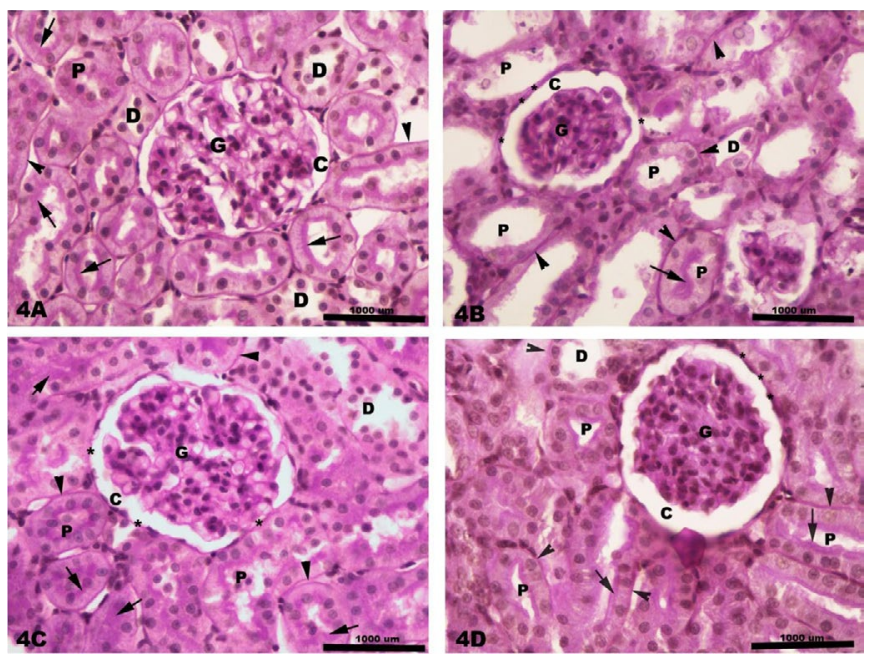

Figure 4: Light photomicrograph of PAS stained sections of rat renal cortex showing $4 \mathrm{~A}$ ) an even positive PAS reaction ditributed within the brush border (arrow) of proximal tubules $(\mathrm{P})$ and in the basal membrane of both proximal and distal (D) tubules (arrow head). As well as, a positive PAS reaction is seen within the outer membrane $\left({ }^{*}\right)$ of the Bowman's capsule $(C)$ in control rats; $4 B$ ) positive PAS-reaction are seen in few proximal tubules $(P)$ that have intact brush border (arrow) and in the basement membrane (arrow head) of both proximal and distal tubules (D) in cisplatin-treated rats. Positive PAS reactions (arrow) are observed in the brush border (arrow) of the proximal tubules $(P)$, in the basal membrane (arrow head) of both proximal and distal (D) tubules and in the outer membrane of the Bowman's capsule $\left({ }^{*}\right)$ in misoprostol-treated rats (4C) and in combined misoprostol+ cisplatin-treated rats. G: glomerulus.

fibers showed no morphological difference in the other three groups (Figure 3B-3D) compared to those of the control rats.

In control rats, a strong positive PAS reaction was observed in brush border and basal membrane of proximal tubules, basement membrane of distal tubules and within the outer glomerular layer (Figure 4A). In CP-treated rats, as most of the brush border was destroyed, a positive PAS reaction was noted only in the remaining brush border of the proximal tubules and basement membrane of both proximal and distal tubules (Figure 4B). In MP-treated rats, a positive PAS reaction was observed in the brush border and basement membrane of proximal tubules, basement membrane of distal tubules and parietal layer of Bowman's capsule (Figure 4C). In combined MP and CP-treated rats, a positive PAS reaction was observed in the regenerated brush border of the proximal tubular cells, the basement membrane of both proximal and distal tubules and outer layer of Bowman's capsule (Figure 4D).

\section{Electron microscopy}

The renal corpuscle of control rats revealed normal ultrastructure (Figure 5A) The podocyte of the inner layer of Bowman's capsule had central intended nucleus surrounded by lighter cytoplasm. This podocyte had thick primary cytoplasmic processes that extended and subdivided into many thin secondary cytoplasmic processes on the outer aspect of the basal lamina of many glomerular capillary loops. Slit diaphragms of the capillary loops were seen between the secondary cytoplasmic processes. In close proximity to the basal lamina of the glomerular capillary, the mesangial cells were seen. These mesangial cells were characterized by small darkly-stained nuclei surrounded by little electron-dense cytoplasm. The glomerular capillary had a continous basal lamina with fenestrated flat endothelial cell-lining. A narrow capsular space was observed between the podocyte and the basal lamina of the capillary tuft (Figure 5A).
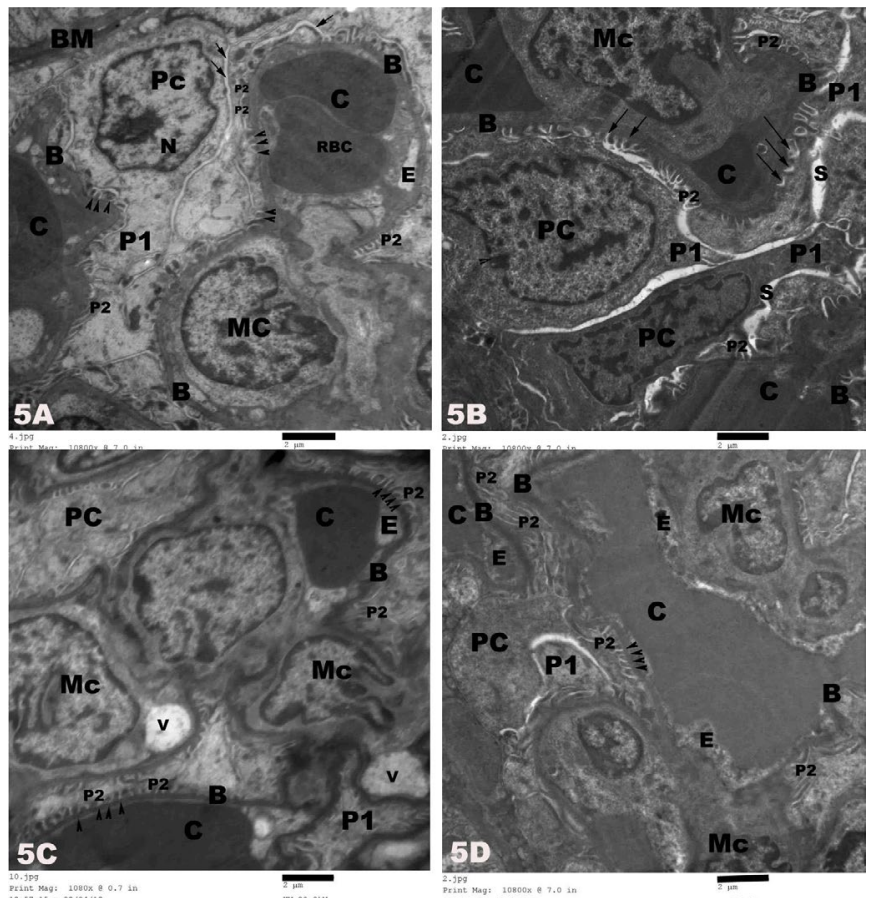

Figure 5: Electron micrographs of rats renal glomeruli showing $5 \mathrm{~A}$ ) the podocyte $(\mathrm{Po})$ of the inner capsular membrane having an irregular nucleus $(N)$, few thick primary cytoplasmic processes (P1) and numerous secondary processes (P2) on the basement membrane (BM) of the glomerular capillary $(\mathrm{Ca})$ with slit diaphragms (arrow heads) in-between. The glomerular capillary is lined by discontinuous endothelial cells $(E)$ and contains blood cells within its wide lumen (RBC). In addition, mesangial cell (Mc), with large nucleus surrounded by little amount of electron-dense cytoplasm, is seen between the capillary loops in the control rats; $5 \mathrm{~B}$ ). in cisplatin-treated rats there are partial fusion of the secondary foot processes (P2) of the podocytes (Pc) on the basement membrane (BM) of the glomerular capillary (Ca), wide capsular space (arrow) and dilation and congestion of between the glomerular capillary (Ca); $5 \mathrm{C}$ ) many mesangial cells (Mc) containing vacuoles (V) are observed in the glomerulus of misoprostoltreated rats; 5D) dilation capillary loops is seen within the glomerulus in combined misoprostol and cisplatin-treated rats group.

The renal corpuscle in CP-treated rats revealed widening of the capsular space, dilation and congestion of the glomerular capillary with fusion of the secondary foot processes of the podocytes in certain areas (Figure 5B). The renal corpuscle of the MP-treated rats showed many mesangial cells in-between the capillary loops. Vaccules of different sizes, shape and contents were seen within the cytoplasm of the mesangial cells (Figure 5C). In combined MP and CP-treated rats, a few mesangial cells and regular distrubtion of the secondary foot processes on normal capillary basement membrane with narrow capsular space were noticed in the renal corpuscle (Figure 5D).

The ultrastructure of the proximal tubular cells in control rats showed numerous long densely packed apical microvilli projecting within the tubular lumen and regular basement cell membrane with many basal infoldings. Basal oval heterochromatic nucleus with central dense nucleolus, surrounded by many mitochondria, lysosomes and apical vacuoles, pinocytotic vesicles and a few singly distributed short rough endoplasmic reticulum cisternae (Figure 6A).

In CP-treated rats, the proximal tubular cells revealed reduction of cell size, number of basal infoldings, apical lysosomes and pinocytotic vesicles with fragmentation and shedding the apical microvilli. In addition, small sized nuclei with irregular nuclear envelope, margination of nuclear heterochromatin with loss of nucleolus, condensation and 
Citation: Nasr AY (2013) Effect of Misoprostol on Ultrastructural Changes of Renal Tissues in Cisplatin-Treated Adult Rats. J Cytol Histol 4: 175. doi:10.4172/2157-7099.1000175

reduction of mitochondrial size were seen in the proximal tubular cells as well (Figure 6B). In MP-treated rats, the proximal tubular cells had oval basal nuclei with peripheral nucleolus. The cell cytoplasm contained many elongated mitochondriae and a few vacuoles and lysosomes. The luminal surface of the cells had long densely packed microvilli (Figure 6C). In combined MP and CP-treated rats, the proximal tubular cells revealed oval euchromatic nucleus with peripheral electron-dense nucleolus, numerous elongated mitochondria, perinuclear electronlucent vacuoles, a few apical lysosomes, many apical pinocytotic vesicles and long microvilli (Figure 6D).

The Distal Tubular Cells (DTC) of the control rats revealed regular trilaminar basement membrane with many basal infoldings, sharp luminal outline and wide lumen. Moreover, numerous basal elongated and round-shaped mitochondria, a few basal lysosomes and short rough endoplasmic reticulum cisternae were seen within their cytoplasm. The cells showed spherical apical nucleus with a central or peripheral electron-dense nucleolus (Figure $7 \mathrm{~A}$ ). In CP-treated rats, the distal tubular cells revealed marked thickening and irregularity of the basement membrane, many basal infoldings. Their cytoplasm contained many elongated mitochondria, a few degenerated mitochondria,
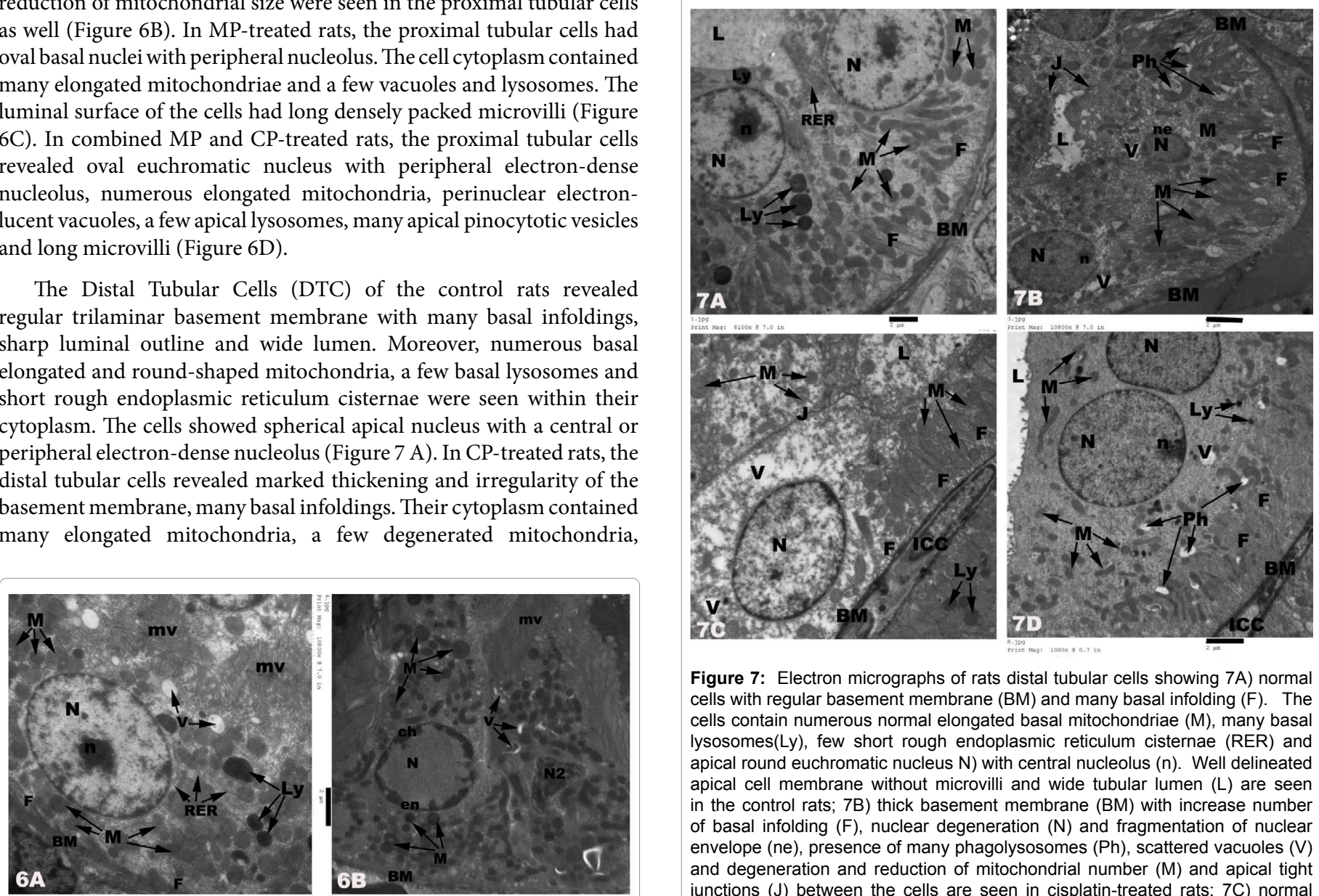

Figure 7: Electron micrographs of rats distal tubular cells showing $7 \mathrm{~A}$ ) normal cells with regular basement membrane $(B M)$ and many basal infolding $(F)$. The cells contain numerous normal elongated basal mitochondriae (M), many basal lysosomes(Ly), few short rough endoplasmic reticulum cisternae (RER) and apical round euchromatic nucleus $\mathrm{N}$ ) with central nucleolus $(\mathrm{n})$. Well delineated apical cell membrane without microvilli and wide tubular lumen $(L)$ are seen in the control rats; 7B) thick basement membrane (BM) with increase number of basal infolding $(F)$, nuclear degeneration $(N)$ and fragmentation of nuclear envelope (ne), presence of many phagolysosomes $(\mathrm{Ph})$, scattered vacuoles $(\mathrm{V})$ and degeneration and reduction of mitochondrial number (M) and apical tight junctions $(\mathrm{J})$ between the cells are seen in cisplatin-treated rats; $7 \mathrm{C}$ ) normal basement membrane (BM) with many basal infoldings $(F)$, many elongated basal mitochondriae $(M)$, central oval euchromatic nucleus $(N)$, few electronlucent vacuoles $(\mathrm{V})$ and apical tight junctions $(\mathrm{J})$ with presence of intercalated cell (ICC) between the distal tubular cells are observed in misoprostol-treated rats; 7D) normal basement membrane (BM) with many basal infoldings (F) and intercalated cell (ICC) are seen in the combined misoprostol and cisplatin-treated rats. Also, numerous elongated normal basal mitochondriae (M), few lysosomes (Ly) and phagosomes (Ph), basal vacuoles (V) and apical round euchromatic nucleus $(\mathrm{N})$ with peripheral nucleolus $(\mathrm{n})$ are seen as well.

many lysosomal vacuoles and phagosomes in close proximity to the mitochondrial membrane. Apoptotic nuclear changes were seen within few cells, where the nucleus showed marked reduction of its size, loss of its nucleolus and heterochromatin with disrupted nuclear envelope (Figure 7B). The distal tubular cells of MP-treated rats showed central oval euchromatic nucleus, numerous basal mitochondria and many electron-lucent vacuolar. Tight apical junctions and longitudinal intercalated cells were observed (Figure $7 \mathrm{C}$ ). In combined MP and $\mathrm{CP}$-treated rats, the distal tubular cells revealed regular many basal infoldings with many elongated mitochondria and a few vesicles inbetween. The cells had apical spherical euchromatic nucleus with peripheral nucleolus (Figure 7D).

\section{Discussion}

$\mathrm{CP}$ is one of the common and effective antineoplastic chemotherapeutic drugs, although its side effects mainly nephrotoxicity, limit its therapeutic uses [2]. The exact underlying mechanism of CPinduced nephrotoxicity is not well known. However, one of the main mechanisms of CP nephrotoxicity is the oxidative stress $[14,15,16]$ 
Oxidative stress leads to the generation of reactive oxygen species (ROS) with suppression of the mitochondrial antioxidants and cellular injury [17].

In the present study, a significant reduction of the body weight, decrease of kidney weight and increase of the kidney weight/body weight ratio were recorded in CP-treated rats compared to control rats. These findings agree with other investigators who reported that the nephrotoxic effect of $\mathrm{CP}$ produced marked reduction of the rats' body weight with a significant increase in the relative kidney weight $[14,18,19]$. Such reduction of the body weight in CP-treated rats might be in part due to gastrointestinal toxicity and concomitant loss of the animal appetite with subsequent reduction of food ingestion [20] or due to excessive loss of water, salts and proteins as a result of renal injury with subsequent dehydration and weight loss [19]. The changes of kidney weight to body weight ratio in CP-treated rats could be due to tissue damage and reduction in their functions [7].

The results of the present study revealed marked elevation of creatinine and BUN levels in CP-treated rats compared to control rats. In agreement with our results, Mansour et al. [14] and Sueishi et al. [15] reported that the nephrotoxic effect of $\mathrm{CP}$ was evidenced by a significant increase of creatinine and BUN levels. The increased creatinine level in CP-treated rats might be due to glomerular damage as a result of ROS generation $[14,15]$. The ROS inhibits the activity of the antioxidant enzymes in renal tissues with increased lipid peroxidation and nephrotoxicity [5].

In agreement with the results of present study, Abdelmeguid et al. [18]; Azu et al. [19]; Ozer et al. [6] reported that the light microscopy of CP-treated rats renal sections revealed marked tubular degeneration, necrosis and desquamation of the tubular epithelial cells with cystic formation, interstitial cellular infiltration, wide capsular space and congested glomerular capillary tufts. The authors added that the oxidative stress was the major pathway of $\mathrm{CP}$ nephrotoxicity. This oxidative stress inhibits the antioxidant enzymes and generates the ROS that destroys the lipid, protein and DNA components of the cell with subsequent enzymatic inactivation and mitochondrial dysfunction. In the present study, the morphological findings of CP-treated rats revealed glomerular and tubular affections. These morphological findings could explain the disturbances of both glomerular and tubular functions, where these disturbances might be the main cause of reduction of body weight, increase the relative kidney weight, elevation of creatinine and BUN levels.

Similar to the findings of the present study, the nephrotoxic effect of $\mathrm{CP}$ was concentrated mainly on the proximal convoluted tubules at the cortico-medullary zone [21], where the necrotic and apoptotic changes of the renal tubular cells are probably due to the direct effect of the intracellular CP $[14,15]$. The accumulation of CP within the cells promotes the generation of the ROS via different pathways [4]. Such ROS might play a role in the pathogenesis of tubular cell apoptosis but probably had no role in the tubular necrosis. Thus, the mechanism of cisplatin-induced renal tubular necrosis was thought to be concentration-dependent [16]. In disagreement with this, massive necrosis and subsequent regeneration of renal proximal tubular cells with minor papillary injury and no glomerular morphological changes were observed in CP-treated rats [22].

Antioxidants were suggested to have a protective role against CP-induced nephrotoxicity mediated by oxidative stress $[14,15,16]$. Therefore, several studies were performed to minimize the nephrotoxic side effect of CP using different antioxidants $[4,18,19,23,16]$. Recently,
MP has gained considerable interest as an ROS scavenger [8] in addition to its antiapoptotic or cytoprotective effects $[9,10]$. The antioxidant effects of MP prevented liver damage by reducing oxygen free radicals after carbon tetra-chloride ( $\mathrm{CCl} 4)$ exposure [8]. The cytoprotective effect of MP was based on to increase mucus and $\mathrm{HCO}^{-3}$ secretion, with a subsequent increase the negative electrical potential of maximal barrier as a result of reduction of the back diffusion of $\mathrm{H}^{+}$, to increase the blood flow of the mucus, to increase the regenerative capacity of the epithelial cells [23]. Moreover, MP blocked the apoptotic changes that were generated through the toxic effect of D-galactosamine [24]. The results of the present study revealed that the oral intake of MP limited the histopathological changes of CP-nephrotoxic changes. Moreover, Asci et al. [25] postulated that, MP had beneficial effects on methotrexate-induced hepatic and renal toxicity in rats.

Asci et al. [25] reported that, the reduction of CP nephrotoxicity by the concomitant use of MP was evidenced by significant reduction of creatinine and BUN levels as well as marked reduction in protein loss in urine. In the present study, co-administration of $\mathrm{MP}+\mathrm{CP}$ improved the histopathological findings of $\mathrm{CP}$-induced renal toxicity, where only mild tubular degenerative changes were noticed in the combined MP and CP-treated rats. The beneficial renoprotective effect of MP in CP-treated rats might be due to its antioxidant and / or cytoprotective, antiapoptotic effects or other unknown mechanism [25,26].

Our study showed marked ultrastructural changes in CP-treated rats compared to control rats. These changes were most prominent in PCT and to a lesser extent in DCT and renal corpuscles. The findings of the present study are in agreement with those of other investigators $[6,18]$.

Moreover, the nephrotoxic effects of $\mathrm{CP}$ were more pronounced at the cortico-medullary zone. This might to be due to the exposure of this zone to a high concentration of $\mathrm{CP}$ through the nutrient blood flow to the kidney with subsequent accumulation of a high percentage of the drug within the cells $[27,28]$. Alternatively, the affection of the tubular cells within the cortico-medullary zone might be due to the cumulative effect of $\mathrm{CP}$ within the renal tissues as the kidneys are the main execratory outlets for either intravenous or intraperitoneal CP [29]. The light and electron microscopic findings of the present study confirmed these explanations.

\section{Conclusion}

This research has provided significant insights into the biochemical and ultrastructural changes of the kidney in the $\mathrm{CP}$-treated rats and the possible protective effect of misoprostol. Co-administration of MP with CP resulted in a reduction of the biochemical and morphological changes of CP-induced nephrotoxicity. Thus, the present study provides promising results about the ameliorative effect of MP on CP-induced nephrotoxicity. The results of the present study could be used to plan an effective strategy for the safe use of the $\mathrm{CP}$ in humans.

Further studies are needed to explain the exact protective mechanism of MP against CP-induced nephrotoxicity and to verify whether co-administration of MP with $\mathrm{CP}$ could affect the antineoplastic action of CP in experimental tumor animal models.

\section{Acknowledgments}

The author thanks Prof. Abdel Moneim M. Osman and Prof. Sherif M. Hassan for their continuous assessment and revision of this manuscript. The autho declares that there are no conflicts of interest. The research received no specific grant from any funding agency in the public, community, or non-for profit sectors. 
Citation: Nasr AY (2013) Effect of Misoprostol on Ultrastructural Changes of Renal Tissues in Cisplatin-Treated Adult Rats. J Cytol Histol 4: 175. doi:10.4172/2157-7099.1000175

\section{References}

1. Hanigan $\mathrm{MH}$, Devarajan $\mathrm{P}$ (2003) Cisplatin nephrotoxicity: molecular mechanisms. Cancer Ther 1: 47-61.

2. Jung M, Hotter G, Viñas JL, Sola A (2009) Cisplatin upregulates mitochondria nitric oxide synthase and peroxynitrite formation to promote renal injury. Toxicol Appl Pharmacol 234: 236-246.

3. Dodiya H, Jain M, Goswami S (2011) Study of urinary biomarkers for nephrotoxicity in Wistar rats. J Pharmacol toxicol 6: 571-579.

4. Ajith TA, Nivitha V, Usha S (2007) Zingiber officinale Roscoe alone and in combination with alpha-tocopherol protect the kidney against cisplatin-induced acute renal failure. Food Chem Toxicol 45: 921-927.

5. An Y, Xin H, Yan W, Zhou X (2011) Amelioration of cisplatin-induced nephrotoxicity by pravastatin in mice. Exp Toxicol Pathol 63: 215-219.

6. Ozer MK, Asci H, Oncu M, Calapoglu M, Savran M, et al. (2011) Effects of misoprostol on cisplatin-induced renal damage in rats. Food Chem Toxicol 49: 1556-1559.

7. Park HR, Ju EJ, Jo SK, Jung U, Kim SH, et al. (2009) Enhanced antitumor efficacy of cisplatin in combination with HemoHIM in tumor-bearing mice. BMC Cancer 9: 85.

8. Salam OM, Sleem AA, Omara EA, Hassan NS (2009) Hepatoprotective effects of misoprostol and silymarin on carbon tetrachloride-induced hepatic damage in rats. Fundam Clin Pharmacol 23: 179-188.

9. Yang H, Majno P, Morel P, Toso C, Triponez F, et al. (2002) Prostaglandin E(1) protects human liver sinusoidal endothelial cell from apoptosis induced by hypoxia reoxygenation. Microvasc Res 64: 94-103.

10. Sostres C, Gargallo CJ, Arroyo MT, Lanas A (2010) Adverse effects of nonsteroidal anti-inflammatory drugs (NSAIDs, aspirin and coxibs) on upper gastrointestinal tract. Best Pract Res Clin Gastroenterol 24: 121-132.

11. Patton CJ and Crouch SR (1977) Spectorophotmetric and kinetics investigations of Berthelot reaction for the determination of Ammonia. Analyt Chem 49: 464469 .

12. Hallett CJ, Cook JG (1971) Reduced nicotinamide adenine dinucleotidecoupled reaction for emergency blood urea estimation. Clin Chim Acta 35: 3337.

13. Bancroft JD and Gamble M (2002) Theory and practice of histological techniques. ( $5^{\text {th }}$ edn), Churchill Livingstone. New York,USA.

14. Mansour MA, Mostafa AM, Nagi MN, Khattab MM, Al-Shabanah OA (2002) Protective effect of aminoguanidine against nephrotoxicity induced by cisplatin in normal rats. Comp Biochem Physiol C Toxicol Pharmacol 132: 123-128.

15. Sueishi K, Mishima K, Makino K, Itoh Y, Tsuruya K, et al. (2002) Protection by a radical scavenger edaravone against cisplatin-induced nephrotoxicity in rats. Eur J Pharmacol 451: 203-208.
16. Uehara T, Yamate J, Torii M, Maruyama T (2011) Comparative nephrotoxicity of Cisplatin and nedaplatin: mechanisms and histopathological characteristics. $J$ Toxicol Pathol 24: 87-94.

17. Santos NAG, Bezerra CSC, Martins NM, Curti C, Bianchi MLP, et al. (2008) Hydroxyl radical scavenger ameliorates cisplatin-induced nephrotoxicity by preventing oxidative stress, redox state unbalance, impairment of energetic metabolism and apoptosis in rat kidney mitochondria. Cancer Chemotherapy Pharmacology 61: 145-155

18. Abdel maguid EN, Hania NC, Noura SA (2010) Protective effect of silymarin on cisplatin-induced nephrotoxicity in rats. Pakistan Journal of Nutrition 9: 624636.

19. Azu OO, Francis IOD, Abraham AO, Crescie CN, Stephen OE, et al. (2010) Protective agent, Kigelia Africana Fruit Extract, against cisplatin-induced kidney oxidant injury in Sprague-Dawley rats. Asian Journal of Pharmaceutical and Clinical Research 3: 84-88.

20. Atessahin A, Yilmaz S, Karahan I, Ceribasi AO, Karaoglu A (2005) Effects of lycopene against cisplatin-induced nephrotoxicity and oxidative stress in rats. Toxicology 212: 116-123

21. Townsend DM, Hanigan MH (2002) Inhibition of gamma-glutamyl transpeptidase or cysteine S-conjugate beta-lyase activity blocks the nephrotoxicity of cisplatin in mice. J Pharmacol Exp Ther 300: 142-148.

22. Nagothu KK, Bhatt R, Kaushal GP, Portilla D (2005) Fibrate prevents cisplatininduced proximal tubule cell death. Kidney Int 68: 2680-2693.

23. Brunton LL, Lazo JS, Parker KL (2006) Goodman and Gilman's the Pharmacological Basis of Therapeutics. Mc Grew-Hill Companies, New York USA.

24. Ranchal I, González R, López-Sánchez LM, Barrera P, López-Cillero P, et al. (2006) The differential effect of PGE1 on D-galactosamine-induced nitrosative stress and cell death in primary culture of human hepatocytes/ Prostaglandins Other Lipid Mediat. 79: 245-259.

25. Asci H, Ozer M K, Calapoglyu M, Savran M, Oncu M, et al. (2011) Effect of misoprostol on methotraxate-induced hepatic and renal damages. J Bio Life Sci 2: 32-37.

26. Tufekci O, Gunes D, Ozogul C, Kolatan E, Altun Z, et al. (2009) Evaluation of the effect of acetyl L-carnitine on experimental cisplatin nephrotoxicity. Exp Chemotherapy 55: 451-459.

27. Pabla N, Murphy RF, Liu K, Dong Z (2009) The copper transporter Ctr contributes to cisplatin uptake by renal tubular cells during cisplatin nephrotoxicity. Am J Physiol Renal Physiol 296: F505-F511.

28. Townsend DM, Tew KD, He L, King JB, Hanigan MH (2009) Role of glutathione $\mathrm{S}$-transferase $\mathrm{Pi}$ in cisplatin-induced nephrotoxicity. Biomed Pharmacother 63 : 79-85.

29. Arany I, Megyesi JK, Kaneto H, Price PM, Safirstein RL (2004) Cisplatininduced cell death is EGFR/src/ERK signaling dependent in mouse proximal tubule cells. Am J Physiol Renal Physiol 287: F543-F549. 